\title{
Digital Support for Healthier Eating Habits Among Patients With Type 2 Diabetes: Protocol for a Randomized Clinical Trial Within Primary Care (HAPPY Trial)
}

Ylva Trolle Lagerros ${ }^{1,2}, \mathrm{MD}, \mathrm{PhD}$; Anna Dahlgren ${ }^{1}$, MSc; Linnea Sjöblom ${ }^{1}, \mathrm{MSc}$; Stephanie E Bonn ${ }^{1}, \mathrm{PhD}$

${ }^{1}$ Clinical Epidemiology Division, Department of Medicine, Solna, Karolinska Institutet, Solna, Sweden

${ }^{2}$ Center for Obesity, Academic Specialist Center, Stockholm Health Services, Stockholm, Sweden

Corresponding Author:

Stephanie E Bonn, $\mathrm{PhD}$

Clinical Epidemiology Division

Department of Medicine, Solna

Karolinska Institutet

Eugeniahemmet T2:02

Karolinska Universitetssjukhuset

Solna, 17176

Sweden

Phone: 46851779173

Email: stephanie.bonn@ki.se

\section{Abstract}

Background: Despite the large impact that dietary habits have in the management of diabetes, few tools for supporting healthy eating habits are available for persons with diabetes.

Objective: The aim of this randomized clinical trial is to evaluate the effect of a 12-week, mobile health (mHealth), app-based intervention promoting healthy eating habits among patients with type 2 diabetes.

Methods: The HAPPY (Healthy eating using APP technologY) trial is a randomized clinical trial with two arms aiming to include 200 patients, 18 years of age or older, with type 2 diabetes. Both women and men are eligible for inclusion. Study participants are randomized 1:1 to an intervention group, where they are instructed to use a smartphone app promoting healthy eating, or to a control group, where they receive standard primary care only, for a period of 12 weeks. Each week a new topic (eg, vegetable intake) is introduced via the app. After an introduction text, the user is given a topic-related activity to perform (eg, eat one additional serving of vegetables per day during that week). The app records daily progress and sends automatic reminders or feedback to the user. Dietary intake, body composition, clinical variables, and biomarkers are measured at baseline and at 3-and 6-month follow-ups. An extensive web-based questionnaire comprising several validated questionnaires assessing a number of lifestyle factors is distributed via email at baseline and at 3-, 6-, and 12-month follow-ups; lifestyle factors include, for example, sleep, physical activity, eating behavior, and health-related quality of life. The effect of the intervention on dietary intake (primary outcome) and on glycated hemoglobin and blood lipid levels, body composition, blood pressure, other lifestyle factors, and overall health (secondary outcomes) will be assessed.

Results: Data collection is ongoing. Recruitment of participants started in January 2019. Findings from the study are expected to be published by the end of 2021 .

Conclusions: Technology development provides new ways to promote and support long-term adherence to healthier eating habits. mHealth-based approaches allow for real-time interaction and the delivery of an intervention at any time. Further, focusing on overall diet allows the user to apply new knowledge to current eating patterns, creating an individualized approach. In this study, we evaluate the effect of using a new smartphone app promoting healthy eating habits on dietary intake, clinical markers, and lifestyle factors among patients with type 2 diabetes.

Trial Registration: ClinicalTrials.gov NCT03784612; https://clinicaltrials.gov/ct2/show/NCT03784612

International Registered Report Identifier (IRRID): DERR1-10.2196/24422

(JMIR Res Protoc 2020;9(11):e24422) doi: 10.2196/24422 


\section{KEYWORDS}

body composition; diabetes; dietary intake; HbA1c; metabolic health; mHealth; obesity; randomized clinical trial; serum lipids; smartphones

\section{Introduction}

\section{Background}

The prevalence of type 2 diabetes is increasing in Sweden, as well as in the rest of the world. Today, over 400 million adults have diabetes [1]. It has long been known that persons with type 2 diabetes have an increased risk of cardiovascular disease (CVD) and premature death, in large part due to an increased prevalence of risk factors for CVD (eg, obesity, dyslipidemia, and hypertension) [2].

More than $80 \%$ of patients with type 2 diabetes in Sweden are overweight or obese [3]; excess adiposity, in particular visceral obesity, as well as insulin resistance are strongly associated with an increased risk of both type 2 diabetes and CVD [4]. A healthy diet is the key factor in both prevention and management of type 2 diabetes. Patterns of vegan, vegetarian, and Mediterranean diets have been shown to improve glycemic control in patients with type 2 diabetes in randomized clinical trials [5]. An unhealthy lifestyle (eg, with poor dietary habits and physical inactivity), on the other hand, increases the risk of developing the disease, as well as the risk of complications [1]. In addition to CVD, complications can be serious and may include kidney failure, blindness, and amputation of lower extremities, and could lead to premature death.

Despite the large impact of dietary habits in the management of type 2 diabetes, few tools for supporting dietary changes and maintenance of a healthy diet are available. One strategy to improve health is regular visits to health clinics [6]. This may not always be a feasible strategy given the large number of patients in need of support. To meet both the needs of the patients and the capacity of the health care system, new strategies must be developed. Mobile health (mHealth) (ie, the use of mobile devices, including smartphones, to promote health) is one way to bridge the gap between what patients need and what health care can offer.

In Sweden, over 90\% of adults own and use a smartphone [7], making an app-based intervention feasible for implementation in the general population of patients with type 2 diabetes. Technology-driven diabetes prevention programs (ie, utilizing, for example, text messages, email, automated phone calls, websites, etc) focusing on diet and/or physical activity have been evaluated in several intervention studies focusing on weight loss with promising results [8]. Michaelides et al [9] also showed that a fully mobile diabetes prevention program including a dietary component could facilitate weight loss and weight maintenance. Further, results from two recent reviews summarizing smartphone apps targeting diet have also shown promising results with regard to dietary intake in particular, although none of the apps had been developed for, or evaluated in, patients with type 2 diabetes specifically [10,11]. Nevertheless, among patients with type 2 diabetes, an automated web-based program to support healthy diet has been shown to improve dietary habits when assessed using a quality dietary score [12], and improvements in adherence to the Mediterranean diet and in diet quality overall have been shown after the use of a smartphone app during a period of 3 months [13]. Thus, mHealth strategies show potential as a tool to promote and support healthy eating habits, including among type 2 diabetes patients.

\section{Aim}

We are conducting a randomized clinical trial called the HAPPY (Healthy eating using APP technologY) trial, which is a 12-week mHealth intervention (ie, includes the use of a smartphone app). The main aim of the trial is to evaluate its effect in promoting and improving healthy eating habits (primary outcome) and in improving levels of glycated hemoglobin $\left(\mathrm{HbA}_{1 \mathrm{c}}\right)$, blood lipids, body composition, and blood pressure, as well as other lifestyle factors and overall health (secondary outcomes) in patients with type 2 diabetes. The aim of this paper is to describe the study design and methodology of the HAPPY trial.

\section{Hypothesis}

Our hypothesis is that participants randomized into the intervention group, who will use the smartphone app, will have improved their dietary habits, cardiovascular risk factors, and other lifestyle factors after 12 weeks of active intervention compared to the control group, who will receive standard care. Further, we hypothesize that improved habits will be maintained after an additional 12 weeks of follow-up.

\section{Methods}

\section{Study Design}

The HAPPY trial is a randomized clinical trial with two arms. The research team behind the design of the smartphone app and the study includes nutritionists, epidemiologists, statisticians, and clinicians. The intervention is described according to the CONSORT (Consolidated Standards of Reporting Trials) statement [14] and the CONSORT-EHEALTH (Electronic and Mobile HEalth Applications and onLine TeleHealth) checklist developed specifically for eHealth and mHealth interventions [15].

Patients with type 2 diabetes who volunteer to participate are recruited from primary health care centers in the center and suburbs of Stockholm. Data collection is performed in collaboration with clinicians and nurses at the centers, where they give a brief introduction of the study to their patients. Information about the study is also available in the waiting rooms of the centers, and patients can contact study personnel directly if they are interested in participating. The included primary care centers differ in size, and the number of eligible patients can vary. Those interested in participating in the study are contacted by phone by study personnel and are given more information. Patients that fulfill inclusion criteria and agree to participate are sent an email with a link to the baseline questionnaire, including a web-based consent form. They are 
thereafter scheduled for a meeting with study personnel. During this meeting, participants sign an additional written informed consent form in order to verify that they fully understand the web-based information. Thereafter, baseline measurements are performed and patients are then randomized 1:1 to the intervention or control group. All participants are followed up after 3, 6, and 12 months.

Baseline and follow-up assessments at 3 and 6 months include an extensive web-based questionnaire including assessment of lifestyle factors; a 4-day food record; clinical measurements of height, weight, waist circumference, blood pressure, and body composition; and blood sampling for measurement of $\mathrm{HbA}_{1 \mathrm{c}}$ and serum lipids. After 12 months, participants are followed up with a final web-based questionnaire. All participants continue to receive usual care by their primary caregiver (ie, they visit their primary caregiver as planned as if they had not been part of the study). Participants randomized to the intervention group use the smartphone app during the 12 weeks of the active intervention from baseline, while participants in the control group will use the app during the 12 weeks after the first follow-up at 3 months. Participants are encouraged to use the app daily, but there is no requirement of how often participants should use the app. The study design is presented in Figure 1.

Figure 1. Study design of the randomized clinical intervention, the HAPPY trial, aiming to evaluate the use of an app-based program for healthy eating habits. HAPPY: Healthy eating using APP technologY; $\mathrm{HbA}_{1 \mathrm{c}}$ : glycated hemoglobin.

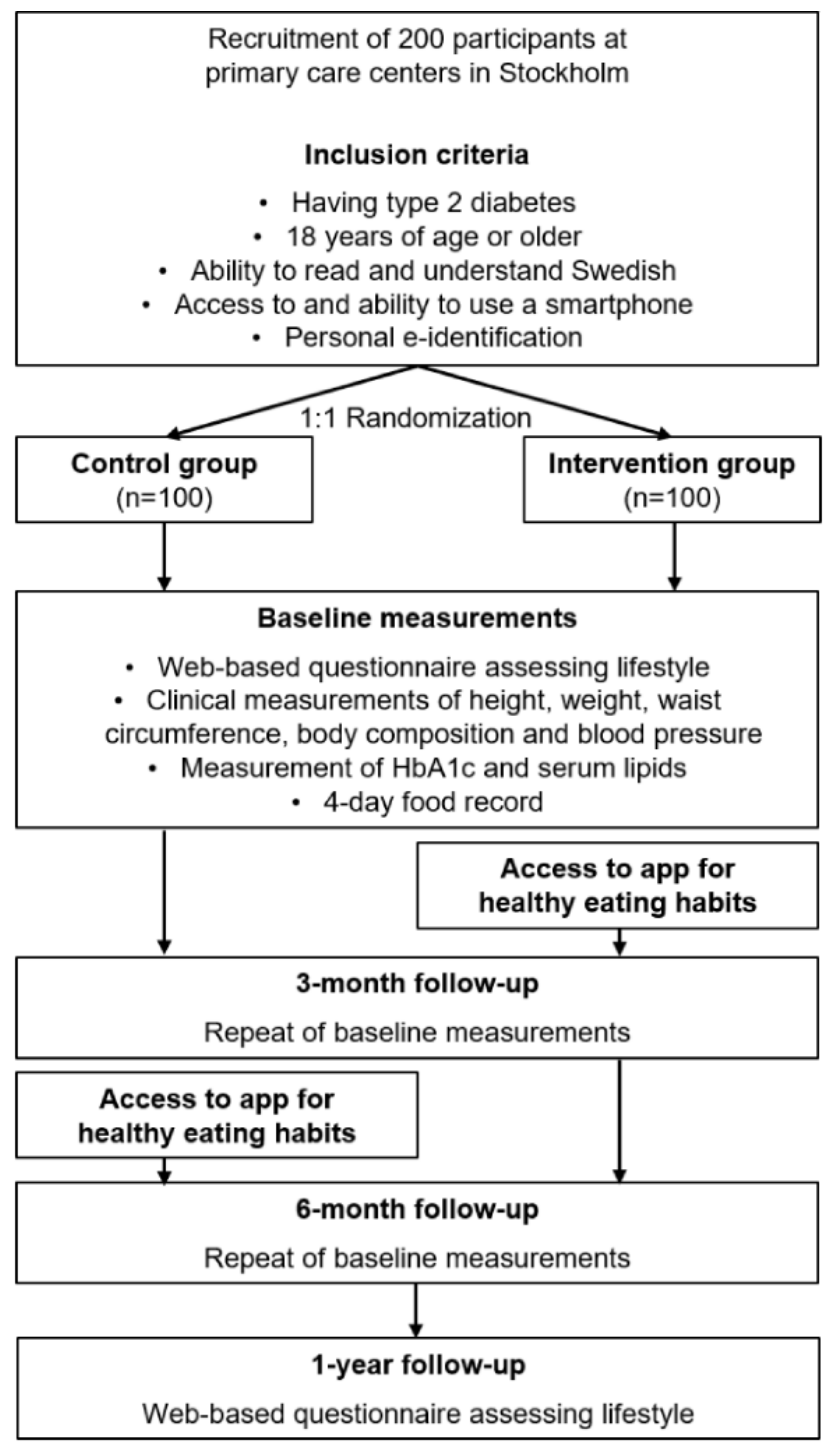

\section{Ethical Approval, Trial Registration, and Consent to Participate}

The trial was approved by the ethics committee of the Regional Ethical Review Board, Stockholm, Sweden (2018/652-31; 2018/1094-32; 2018/2393-32). The trial was registered at ClinicalTrials.gov (NCT03784612). All study participants receive oral and written information about the study and give their written informed consent prior to study start.

\section{Inclusion and Exclusion Criteria}

Inclusion criteria are having type 2 diabetes diagnosed by a physician; being 18 years of age or older; having the ability to read and understand Swedish; having access to, and the ability to use, a smartphone; having a personal e-identification (ie, a secure, digital citizen e-identification solution to enable personal and secure identification in the app); and giving informed consent for participation. Both women and men are eligible for inclusion. No specific exclusion criteria apply. 


\section{Randomization and Blinding}

Study participants are randomized to either the intervention group (ie, standard care and use of the smartphone app at study start) or the wait-listed control group (ie, standard care and use of the smartphone app after 3 months). Randomization is done by study personnel (AD and LS) at baseline at a 1:1 ratio using an allocation sequence list generated in Stata, version 14.0 (StataCorp LP). Women and men are randomized separately in blocks of 4 within each participating primary care center in order to assure an even distribution between the intervention and control groups. Due to the nature of the intervention, participants are not blinded to their allocation.

\section{Intervention}

\section{Development and Content of the HAPPY Smartphone App}

The primary aim of the intervention is to achieve an improvement in dietary intake and subsequent improvements in clinical variables and lifestyle factors through the use of a smartphone app for healthy eating habits among patients with type 2 diabetes. The content of the HAPPY smartphone app and the healthy eating behavior program is built into an existing digital platform developed by FRISQ AB. The program with its contents has been developed by researchers with experience in behavior change interventions (eg, physical activity), nutritionists, and active clinicians within primary and specialist care. It is based on a theoretical framework including the health belief model and the stages of change model, as well as on social cognitive theory [16]. Several techniques for behavior change, including general information, goal-setting strategies, self-monitoring, and feedback on performance, are included in the program [17]. The specific features of the HAPPY smartphone app are described below. The app is available both for iOS (version 11.4 and higher) and Android (version 5.0 and higher).

\section{The HAPPY Smartphone App}

Each week the user will be introduced to a new topic on healthy eating habits (eg, vegetable intake). The specific topics for each of the 12 weeks of the active intervention are outlined in Table 1. Following a short written introduction to the current topic, the user is given an activity to perform (eg, to add one portion of vegetables per day during that week or replace sugar-sweetened beverages with water during a week). Daily progress is recorded in the app, and automatic reminders (eg, notifications if the user has not responded to an activity) or feedback on the activity (eg, "Thank you for participating in the activity") will be given. All study participants receive the same introduction and activity to perform. Reminders and feedback depend on the actions taken by the user.

Table 1. Topics of the healthy eating program for each week of the intervention.

\begin{tabular}{|c|c|}
\hline Week & Topic \\
\hline 1 & Healthy food patterns \\
\hline 2 & Vegetable intake \\
\hline 3 & Regular eating habits \\
\hline 4 & Sugar intake \\
\hline 5 & More on vegetable intake \\
\hline 6 & Slow and fast carbohydrates \\
\hline 7 & Whole grains and fibers \\
\hline 8 & Legumes \\
\hline 9 & Saturated fat \\
\hline 10 & Unsaturated fat \\
\hline 11 & Salt intake \\
\hline 12 & Beverages \\
\hline
\end{tabular}

Each topic (ie, each week) comprises information, recipes, short fun facts, or advice (ie, edutainment and an activity related to the topic). Every Monday, information and an activity that is linked to the theme of the week are available in the app. Recipes to inspire healthy cooking and facts or advice on healthy eating habits are introduced during the week on Tuesday, Wednesday, Friday, and Saturday. Every Thursday, the user receives a reminder of the activity, and at the end of the week, an evaluation about the activity is sent to the user. Each action (ie, reading the information text, recipes, short facts, or advice, and then performing the activity) is marked as read or completed in the app by the user. The status of an action in the app (ie, read vs not read or completed vs not completed) is also visible to study personnel. This information, together with information on each time a user has logged on to the app, is saved within the system. The structure of one week in the healthy eating program is presented in Table 2. Examples from the HAPPY smartphone app are shown in Figure 2. 
Table 2. Structure of weekly activities in the app.

\begin{tabular}{ll}
\hline Day & Content \\
\hline Monday & Information and activity introduction \\
Tuesday & Recipe 1 and recipe 2 \\
Wednesday & Short fact or advice 1 \\
Thursday & Activity reminder: "How is it going?" \\
Friday & Recipe 3, recipe 4, and recipe 5 \\
Saturday & Short fact or advice 2 and short fact or advice 3 \\
Sunday & Activity evaluation: "How did it go?" \\
\hline
\end{tabular}

Figure 2. Examples of screenshots from the HAPPY smartphone app. a) Each day, the specific activities for that day are displayed to the user. Before completing an activity, $0 \%$ of the activities for that specific day have been performed, as indicated by the large circle. By touching the screen where an activity is listed, the user is taken to a new page showing the activity in question. When an activity has been completed, this is indicated in the large circle as well as to the left of the activity in the list below the circle. b) At the bottom of the first page in the app, the user can choose the document symbol to display information (left-hand image), recipes (middle image), or short facts and advice (right-hand image). The user can access the documents at any time; for example, they can return to reread information or find favorite recipes. HAPPY: Healthy eating using APP technologY.

a)

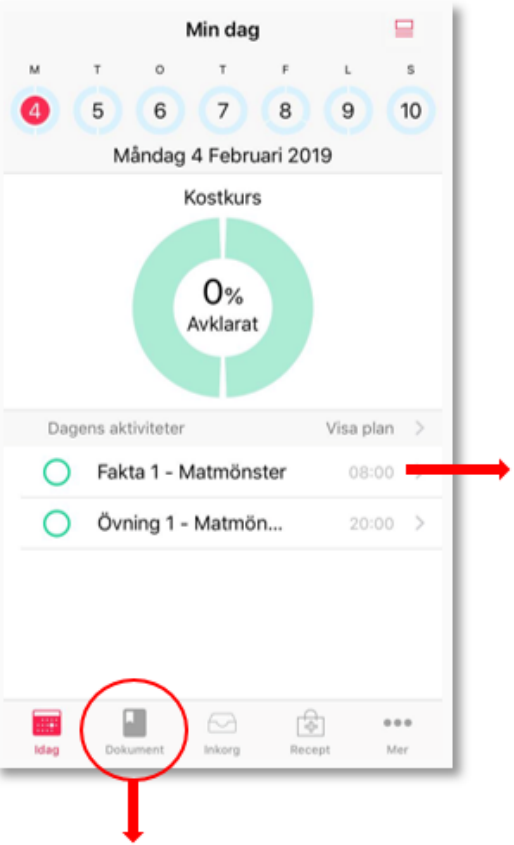

b)

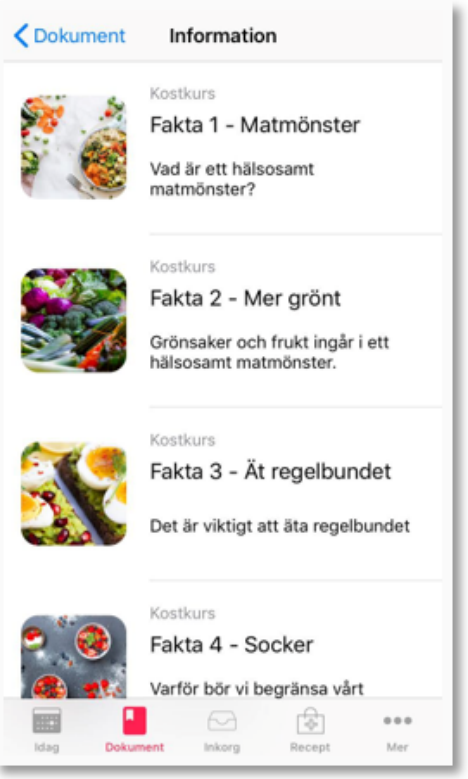

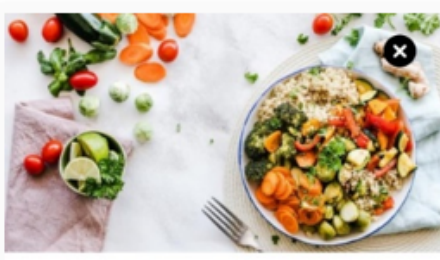

kostkurs

Fakta 1 - Matmönster

Vad är ett hälsosamt matmönster?

Har du sett matpyramiden? Den visar vilka och hur mycket av olika livsmedel som ingár i ett hälsosamt matmönster. Det som finns i botten av pyramiden kan du äta mer av. Dăr finns grönsaker, baljväxter (ärter, bönor, linser), rotfrukter, lök, färska kryddor, frukt, bär, nötter, mandel, frön, grovt bröd och andra fiberrika spannmál samt pasta och ris. Basfettet àr vegetabiliska oljor.
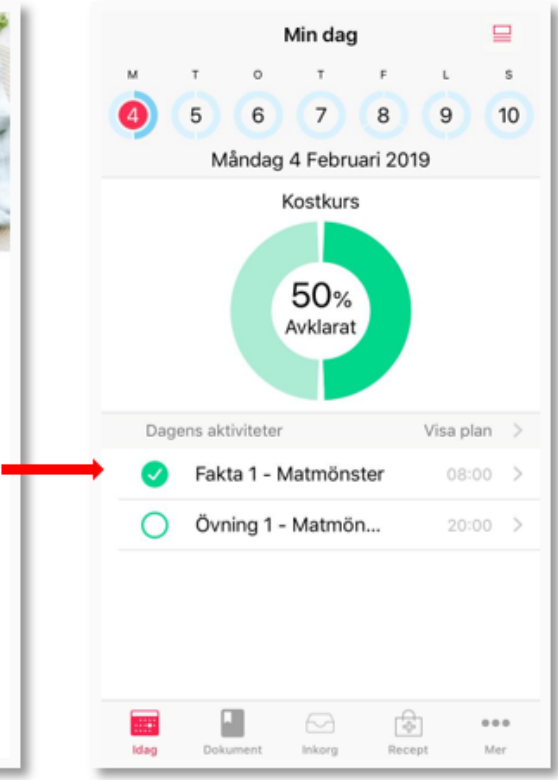

Recept Kostkurs 1.1 Grillad kyckling med s... Perfekt snabbmat năr man har bráttom.

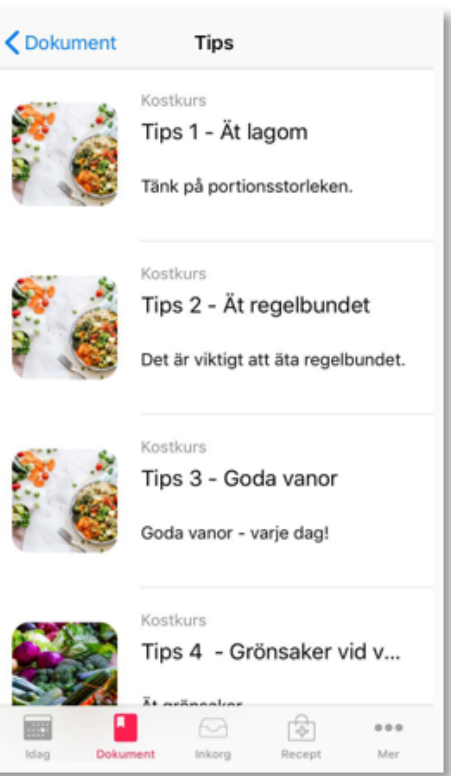




\section{Download}

At baseline, participants in the intervention group will download and be connected to the digital platform, where the study personnel (ie, trained nutritionists) can follow the individual progress of the user during the 12-week course on healthy eating habits in the app. Participants in the control group will be offered to download and be connected to the app at the 3-month follow-up. To avoid overlap with the 4-day food record, the app will be activated on the first Monday following the meeting with study personnel (ie, at baseline or the 3-month follow-up) and the intervention will thereafter follow for 12 consecutive weeks. An individual user account on the digital platform will be created for each participant by study personnel. Users will identify themselves in the app using a personal e-identification

\section{Sample Size and Power Calculations}

A total of 168 patients ( 84 per group) will provide $80 \%$ power at a $5 \%$ significance level to detect a clinically significant change of $4 \mathrm{mmol} / \mathrm{mol}$ in $\mathrm{HbA}_{1 \mathrm{c}}$ [18]. A standard deviation of $11.8 \mathrm{mmol} / \mathrm{mol}$ was estimated based on the average $\mathrm{HbA}_{1 \mathrm{c}}$ level (mean $53.4 \mathrm{mmol} / \mathrm{mol}, 95 \%$ CI 53.3-53.5) in Stockholm County in 2016 using data from the National Diabetes Register [19]. Based on earlier intervention studies within similar populations, a dropout rate of around $20 \%$ is expected. To cover for potential dropout, we will recruit a total of 200 patients (100 per group).

\section{Outcome Measures}

\section{Overview}

A web-based questionnaire including assessment of lifestyle factors; a 4-day food record; clinical measurements of height, weight, waist circumference, blood pressure, and body composition; and blood sampling for measurement of $\mathrm{HbA}_{1 \mathrm{c}}$ and serum lipids will be given to all participants at baseline and at follow-up assessments at 3 and 6 months. At the 12-month follow-up, participants will respond to a final web-based questionnaire.

\section{Dietary Intake From the 4-Day Food Record}

Dietary intake is measured using a 4-day food record over 4 consecutive days, including at least one weekend day. Participants are given a paper diary and instructed by the study personnel, who are trained nutritionists, to write down everything they eat and drink during the period of recording. Type of meal (ie, breakfast, snack, lunch, dinner, etc) is also recorded. The estimated amount of food and beverages consumed can be recorded in different units, including number of items (eg, number of potatoes), weight (eg, $125 \mathrm{~g}$ chicken), or unit of volume (eg, $2 \mathrm{dL}$ of milk or 1 cup of coffee). The participants are requested to maintain their usual diet during the days of recording. The software program Dietist Net (Kost och Näringsdata $\mathrm{AB}$ ) is used by study nutritionists to calculate nutrient intake from the food records; the nutritionists also check the dietary recordings for completeness when they are returned. In the event of items being recorded in an unspecified way (eg, "fish" or "yogurt" without further specification), information on the most commonly consumed fish or yogurt is obtained from nationwide data [20] and entered into the nutrient calculations. Standard portion sizes available for each food item in the Dietist Net software are used if the amount of food was not specified.

The participants complete the food records, as well as respond to a food frequency questionnaire (FFQ), at baseline and follow-up after 3 and 6 months. While the food records will give detailed information on types of food items, portion sizes, and frequency and timing of intake during the day, data from the FFQs allow for comparison to other studies as well as to follow-up of study participants in this study after 12 months.

\section{Body Composition}

Weight, height, waist circumference, and body composition are measured by study personnel at baseline and at 3- and 6-month follow-ups. Weight is measured to the nearest $0.1 \mathrm{~kg}$ in light clothing without shoes, and height is measured to the nearest $\mathrm{cm}$ in a standing position. Waist circumference is measured around the waist, approximately $2 \mathrm{~cm}$ above the umbilicus, to the nearest $\mathrm{cm}$. Body weight and waist circumference are measured once on each occasion. Body composition, including body fat and fat-free mass, is measured using a digital body composition analyzer, Model BC-418 (Tanita). The scale utilizes an 8-electrode bioelectrical impedance analysis with current going from foot to hand and from hand to foot on both sides of the body.

\section{Blood Pressure}

Blood pressure - systolic and diastolic-is measured by the study personnel using the M7 Intelli IT automatic electronic monitor with Bluetooth technology (Omron). Measurements are done with each participant in a seated position with legs uncrossed after the participant has been sitting for at least 5 minutes.

\section{Biomarkers}

Biomarkers are measured in fasting blood samples at baseline and at 3-and 6-month follow-ups. Study participants visit their closest primary care unit to have the blood samples taken. All samples are sent for analysis to the same lab connected to the Karolinska University Hospital in Stockholm, Sweden. HbA $(\mathrm{mmol} / \mathrm{mol})$ is measured using the IFCC (International Federation for Clinical Chemistry and Laboratory Medicine) reference measurement procedure [21,22]. Triglycerides (mmol/L), total cholesterol ( $\mathrm{mmol} / \mathrm{L})$, and high-density lipoprotein (HDL) cholesterol (mmol/L) are measured using the enzymatic method. Low-density lipoprotein (LDL) cholesterol $(\mathrm{mmol} / \mathrm{L})$ is calculated using the Friedewald equation:

$$
\begin{aligned}
& \text { LDL cholesterol }=\text { total cholesterol }- \text { HDL cholesterol } \\
& -(0.45 \times \text { triglycerides })
\end{aligned}
$$

The ratio of LDL cholesterol to HDL cholesterol is calculated from the levels of these lipoproteins.

\section{Web-Based Questionnaire}

Study participants respond to a web-based questionnaire at baseline and at follow-up after 3, 6, and 12 months. A link to the questionnaire is emailed to study participants on each occasion. A first reminder is sent if the participant has not 
responded within 1 week, and an additional reminder is sent 1 week after that if there is still no response. Each questionnaire takes approximately 30-45 minutes to complete and is comprised of several different sections as specified below. If not otherwise specified, the web-based questionnaire is comprised of the questions below.

Background information on marital status, education, and tobacco use (ie, smoking and snuff use) is assessed. Participants are also asked to report the year that they were diagnosed with type 2 diabetes; medication use related to hypertension, hyperlipidemia, and diabetes (ie, insulin or other treatment); and if they have changed their medication during the past 30 days. Background information is only collected from the baseline questionnaire.

Dietary intake is assessed using a 94-item semiquantitative FFQ. The FFQ has been validated previously in a random sample of Swedish men [23]. Spearman correlation coefficients for macronutrients comparing intake assessed using the FFQ, for an average of 14 24-hour recalls spread out over 1 year, were .44 (protein), .70 (total fat), .73 (carbohydrates), and .81 (alcohol). Participants report how often, on average, they consume each included food and beverage, including alcohol. An additional six questions developed by the Swedish National Food Agency, that are used in clinical practice, assessing overall dietary habits and risk use of alcohol are also included [24].

Eating behavior is assessed using the 21-item Three-Factor Eating Questionnaire [25]. The questionnaire includes questions related to cognitive restraint (six questions), uncontrolled eating (nine questions), and emotional eating (six questions).

Physical activity and sedentary behavior are assessed using general questions commonly used in clinical practice, regarding time spent doing physical activities at a moderate-intensity level or higher and sitting time [24], using the Active-Q questionnaire [26,27]. Active-Q includes 48 items and assesses habitual activity in four different domains: daily occupation, transportation, leisure time, and regular sports activities.

Health-related quality of life is assessed using the RAND-36, a questionnaire developed by the RAND Corporation [28]. RAND-36 comprises 36 questions within eight domains: physical functioning, role limitations due to physical health, role limitations due to emotional problems, energy and fatigue, emotional well-being, social functioning, pain, and general health. Responses from the individual domains are summarized into an overall physical component summary score and an overall mental component summary score. Additionally, a Swedish translation of the Life Engagement Test comprised of six questions is used to assess purpose in life [29].

Sleeping habits are assessed using a 13-item version of the Karolinska Sleep Questionnaire [30,31]. Participants report the time of going to bed the previous evening, time of waking up in the morning thereafter, and time from going to bed to falling asleep. They are also asked to report on sleep quality.

Stress level is assessed using a 14-item version of the Perceived Stress Scale developed by Cohen et al [32]. Participants are asked to respond to how often they perceive themselves to react

http://www.researchprotocols.org/2020/11/e24422/ in different, potentially stressful situations on a 5-point Likert scale, ranging from "Never" to "Very often."

Diabetes self-efficacy and distress are assessed using the 20-item Swedish translation of the Problem Area in Diabetes Questionnaire [33]. Participants are asked to rate their distress with having diabetes on a 5-point Likert scale, ranging from "Not a problem" to "A serious problem."

Perceivedsocialsupport for healthy eating habits is assessed by three questions regarding general support and support from friends and family. The questions were developed for this study and were based on the Physical Activity Social Support Scale [34]. Participants are asked to rate the statements "I experience support and encouragement to eat healthy food from people around me," "...my friends," and "...my family" using four response alternatives, ranging from "Agree completely" to "Do not agree at all."

Evaluation questions about the usability of the app [35] and the contents of the healthy eating program are included in the 3-month follow-up questionnaire for participants in the intervention group and in the 6-month follow-up questionnaire for participants in the control group. This is done so that participants respond to the evaluation directly after having used the app. In total, users are asked to respond to 12 statements about the usability of the app (eg, "The app was easy to use") and 12 statements regarding the content in the healthy eating program (eg, "The healthy eating program has made me reflect upon my dietary habits") using a 5-point scale, ranging from "Do not agree at all" to "Completely agree." In addition, users are also asked to leave a free-text comment regarding (1) what was good about the app and (2) what could be improved.

\section{Statistical Analysis}

All data from clinical assessments, the questionnaires, and the app are anonymized and continuously stored at secure servers. Descriptive statistics will be summarized to describe participant characteristics at baseline and follow-up. Data will be checked for outliers and normality. Results will be stratified by control and intervention group. Baseline results will be tested to assess the success of the randomization in balancing characteristics. Differences between groups will be assessed using Student $t$ tests, analyses of variance, and logistic regression. Any detected differences in baseline characteristics between the intervention and control groups will be taken into account as potential confounders in further analysis.

Testing for trends over time in outcomes will be conducted using statistical methods for longitudinal data. Generalized estimation equations will be used to assess the effect of both time and the intervention itself on outcomes. We will control for any unbalanced baseline characteristics. Testing for interactions will be conducted in the models, and intention-to-treat analysis will be performed to account for effects of crossover and dropout. Sensitivity analysis to account for missing data will be done. To further study if the effect of the intervention differs based on participant characteristics, stratified analysis will be performed.

Lastly, we will analyze user satisfaction and user statistics, including number and length of visits to the platform via the 
app. The number of completed actions during the active intervention will also be analyzed to assess user engagement with the app. Associations between use of the platform and characteristics of participants will be investigated.

\section{Results}

The first study participants were recruited in January 2019. Data collection is ongoing. Recruitment is planned to continue until a total of 200 study participants have been included or up to the end of June 2021, whichever comes first. Data collection, including follow-up assessments, will be complete 1 year after recruitment of the last study participant. We expect to publish findings from the study by the end of 2021 .

\section{Discussion}

In the HAPPY trial, we aim to evaluate the effect of an mHealth intervention (ie, use of a smartphone app) promoting healthy eating habits in patients with type 2 diabetes. We know that a healthy diet is a key factor in the management of type 2 diabetes. However, implementing new habits (eg, healthier eating habits) and maintaining them is difficult. Nevertheless, interventions focusing on promoting healthier eating have been shown to lead to improvements in food habits [36]. Focusing on diet overall, instead of, for example, specific nutrients or energy intake, allows the individual to modify their existing dietary habits according to new advice, increasing the chance of successfully implementing new habits [37].

In a comprehensive review article by Ley et al [37], the authors concluded that the overall diet quality should be emphasized in dietary recommendations to patients. This was based on the gathered evidence showing that a higher intake of whole grains, fruits and vegetables, legumes, and nuts; moderate alcohol consumption; and a lower intake of refined grains, red and processed meats, and sugar-sweetened beverages were associated with a reduced risk of diabetes, as well as improved glycemic control and blood lipid levels in patients with type 2 diabetes. These results have also been supported in a later review [38]. The mHealth smartphone app evaluated in the HAPPY trial focuses on healthy eating habits in general, rather than the energy intake of specific nutrients.

Previous mobile- or app-based intervention studies targeting dietary intake or eating behavior have been summarized in two recent reviews, showing promising results [10,11]. Schoeppe et al [10] summarized results from studies using app strategies to target different lifestyle behaviors, including diet. Most apps targeting diet in adults were shown to be successful in improving dietary intake. Several of the included studies showed an increased fruit and/or vegetable intake or a decreased intake of sugar-sweetened beverages in the intervention groups compared to the control groups. The average duration of the intervention studies included in the review was 10 weeks (range 1-24). Further, Mandracchia et al [11] found that mHealth apps using self-monitoring were effective in increasing fruit and vegetable intake among adults or young adults with overweight. However, none of the apps targeting diet or eating behavior that were included in the two above reviews had been developed for, or evaluated among, patients with type 2 diabetes specifically.

In a study by Holmen et al [39], the authors showed good feasibility of using a mobile phone-based self-management system, including a dietary component, among patients with type 2 diabetes. After 1 year of using a mobile phone-based self-management system, between $30 \%$ and $40 \%$ of the individuals randomized to one of two groups using the system were substantial users of the app (eg, they had at least 50 interactions with the app during the past 6 months). Using an automated web-based program to support healthy diet has also been shown to improve dietary habits among this group of patients [12]. Further, a recent randomized controlled trial including patients with type 2 diabetes showed improvement in adherence to the Mediterranean diet and in diet quality overall after the use of a smartphone app over 3 months [13].

Noteworthy strengths of the HAPPY trial include the randomized design, a large sample size, objective assessment of outcomes (eg, measured clinical and anthropometric variables), and a priori calculation of statistical power. Further, the content of the HAPPY smartphone app was developed in collaboration with researchers, clinicians from primary care, and specialists from endocrinology clinics. The digital platform allows the patient and care provider to work together. This increases the chance of effectiveness, as interventions including both the health care system and patient have been found to be more effective than those targeting only one or the other [40]. Nevertheless, a limitation to our study is the lack of a pilot study testing feasibility and acceptability of the app among users. However, the healthy eating program is built into an existing digital solution that has been rigorously tested and is continuously updated to allow for the rapid development of iOS and Android systems. The stability of the digital solution is a strength, as users are less likely to stop using the app due to technical malfunctions. The use of various behavioral change techniques from different theoretical domains may also increase user engagement and decrease attrition in our study. Within the HAPPY trial, we are also collecting data on user engagement with, and usability of, the app and the healthy eating program.

A 4-day food record is used to assess dietary intake at baseline and follow-up. Although most dietary assessment methods are subjective and susceptible to social desirability, a food record has the advantages of being prospective (ie, does not rely on memory) and open ended with no limitation on what items can be reported. In the event of potential missing data in the food records (ie, unspecified food items being recorded or information on portion size is missing), this information will be added using data from a nationwide study of dietary intake in Sweden [20] or replaced by a standard portion size. This may affect results from the food records. However, such missing information is likely random. To enable long-term follow-up of dietary intake as well as comparison to other studies, participants also respond to an FFQ at baseline and follow-up after 3, 6, and 12 months.

Recruitment of study participants is performed at a number of different primary care centers located in areas with different populations and socioeconomic statuses. This increases the generalizability of our results to different groups of patients. 
However, the inclusion criteria of being able to communicate in Swedish and having access to a smartphone may be a limitation of the study. Type 2 diabetes is more common with increasing age. This could be a limitation, as knowledge of how to use a smartphone may be limited among older individuals, leading to a younger study population. Nevertheless, over $75 \%$ of individuals aged 66-75 years in Sweden use the internet on their phone [7]. While patients with limited knowledge of Swedish are more prevalent in areas of lower socioeconomic status, smartphone usage in Sweden is independent of socioeconomic status, and over $90 \%$ of all adults in Sweden own and use a smartphone [7]. The smartphone app was developed for usage on both Android and iOS devices, meaning that most smartphone users can download and use it.
To conclude, the app-based mHealth solution evaluated in the described randomized clinical trial has been developed taking into account the needs of patients, who request mHealth solutions to support a healthy lifestyle, as well as the health care system, which is in need of new, feasible solutions to meet the needs of increasing numbers of patients. Using mHealth strategies, real-time interaction with users is possible and health interventions can be delivered at any time. Further, focusing on diet quality, rather than on specific nutrients, food items, or energy intake, allows the user to apply new knowledge regarding healthy eating habits to his or her current eating patterns, as well as personal and cultural food preferences [41]. Such an individualized approach could be a key factor in promoting and supporting long-term adherence to healthier eating habits among persons with type 2 diabetes.

\section{Acknowledgments}

We would like to thank the personnel at participating primary care centers for their hard work in recruiting study participants. This work is supported by the Strategic Research Area Health Care Sciences (SFO-V) at Karolinska Institutet (SB) and by funds from the regional agreement between Stockholm County Council and Karolinska Institutet (AD, LS, and YTL). The funders had no role in the design of the study, nor have they any role in the data collection, analysis, or interpretation, or in writing of this manuscript.

\section{Conflicts of Interest}

Non declared.

\section{References}

1. World Health Organization. Global Report on Diabetes. Geneva, Switzerland: World Health Organization; 2016. URL: https://apps.who.int/iris/bitstream/handle/10665/204871/9789241565257 eng.pdf?sequence=1 [accessed 2020-03-10]

2. Gu K, Cowie CC, Harris MI. Diabetes and decline in heart disease mortality in US adults. JAMA 1999 Apr 14;281(14):1291-1297. [doi: 10.1001/jama.281.14.1291] [Medline: 10208144]

3. Gubjornsdottir S, Svensson A, Eliasson B, Eeg-Olofsson K, Björck S, Linder E, et al. Årsrapport 2018 [Report in Swedish]. Nationella Diabetesregistret (NDR). URL: https://www.ndr.nu/pdfs/Arsrapport NDR 2018.pdf [accessed 2020-03-10]

4. Yu E, Rimm E, Qi L, Rexrode K, Albert CM, Sun Q, et al. Diet, lifestyle, biomarkers, genetic factors, and risk of cardiovascular disease in the nurses' health studies. Am J Public Health 2016 Sep;106(9):1616-1623. [doi: 10.2105/AJPH.2016.303316] [Medline: 27459449]

5. Papamichou D, Panagiotakos DB, Itsiopoulos C. Dietary patterns and management of type 2 diabetes: A systematic review of randomised clinical trials. Nutr Metab Cardiovasc Dis 2019 Jun;29(6):531-543. [doi: 10.1016/j.numecd.2019.02.004] [Medline: $\underline{\text { 30952576] }}$

6. Appel LJ, Clark JM, Yeh H, Wang N, Coughlin JW, Daumit G, et al. Comparative effectiveness of weight-loss interventions in clinical practice. N Engl J Med 2011 Nov 24;365(21):1959-1968 [FREE Full text] [doi: 10.1056/NEJMoa1108660] [Medline: 22085317]

7. Davidsson P, Palm M, Melin Mandre A. The Swedes and the Internet 2018 [Report in Swedish]. Stockholm, Sweden: Internetstiftelsen; 2018. URL: https://internetstiftelsen.se/docs/Svenskarna och internet 2018.pdf [accessed 2020-03-10]

8. Van Rhoon L, Byrne M, Morrissey E, Murphy J, McSharry J. A systematic review of the behaviour change techniques and digital features in technology-driven type 2 diabetes prevention interventions. Digit Health 2020;6:1-27 [FREE Full text] [doi: 10.1177/2055207620914427] [Medline: 32269830]

9. Michaelides A, Major J, Pienkosz E, Wood M, Kim Y, Toro-Ramos T. Usefulness of a novel mobile diabetes prevention program delivery platform with human coaching: 65-week observational follow-up. JMIR Mhealth Uhealth 2018 May 03;6(5):e93 [FREE Full text] [doi: 10.2196/mhealth.9161] [Medline: 29724709]

10. Schoeppe S, Alley S, Van Lippevelde W, Bray NA, Williams SL, Duncan MJ, et al. Efficacy of interventions that use apps to improve diet, physical activity and sedentary behaviour: A systematic review. Int J Behav Nutr Phys Act 2016 Dec 07;13(1):127 [FREE Full text] [doi: 10.1186/s12966-016-0454-y] [Medline: 27927218]

11. Mandracchia F, Llauradó E, Tarro L, Del Bas JM, Valls RM, Pedret A, et al. Potential use of mobile phone applications for self-monitoring and increasing daily fruit and vegetable consumption: A systematized review. Nutrients 2019 Mar 22;11(3):686 [FREE Full text] [doi: 10.3390/nu11030686] [Medline: 30909484]

12. Hansel B, Giral P, Gambotti L, Lafourcade A, Peres G, Filipecki C, et al. A fully automated web-based program improves lifestyle habits and HbA1c in patients with type 2 diabetes and abdominal obesity: Randomized trial of patient e-coaching 
nutritional support (the ANODE Study). J Med Internet Res 2017 Nov 08;19(11):e360 [FREE Full text] [doi: 10.2196/jmir.7947] [Medline: 29117929]

13. Alonso-Domínguez R, García-Ortiz L, Patino-Alonso MC, Sánchez-Aguadero N, Gómez-Marcos MA, Recio-Rodríguez JI. Effectiveness of a multifactorial intervention in increasing adherence to the Mediterranean diet among patients with diabetes mellitus type 2: A controlled and randomized study (EMID Study). Nutrients 2019 Jan 14;11(1):162 [FREE Full text] [doi: 10.3390/nu11010162] [Medline: $\underline{30646500]}$

14. Schulz KF, Altman DG, Moher D, CONSORT Group. CONSORT 2010 statement: Updated guidelines for reporting parallel group randomised trials. BMJ 2010 Mar 23;340:c332 [FREE Full text] [doi: 10.1136/bmj.c332] [Medline: 20332509]

15. Eysenbach G, CONSORT-EHEALTH Group. CONSORT-EHEALTH: Improving and standardizing evaluation reports of web-based and mobile health interventions. J Med Internet Res 2011 Dec 31;13(4):e126 [FREE Full text] [doi: 10.2196/jmir.1923] [Medline: 22209829]

16. Rimer B, Glanz K. Theory at a Glance: A Guide for Health Promotion Practice (Second Edition). Washington, DC: US Department of Health and Human Services, National Institutes of Health; 2005 Sep. URL: https://cancercontrol.cancer.gov/ sites/default/files/2020-06/theory.pdf [accessed 2020-11-04]

17. Michie S, Richardson M, Johnston M, Abraham C, Francis J, Hardeman W, et al. The behavior change technique taxonomy (v1) of 93 hierarchically clustered techniques: Building an international consensus for the reporting of behavior change interventions. Ann Behav Med 2013 Aug;46(1):81-95. [doi: 10.1007/s12160-013-9486-6] [Medline: 23512568]

18. Berne C, Fritz T. Diabetes mellitus. Läkemedelsboken. Uppsala, Sweden: Läkemedelsverket; 2017. URL: https:/ /lakemedelsboken.se/kapitel/endokrinologi/diabetes_mellitus.html\#k1_10 [accessed 2020-03-10]

19. About NDR. The Swedish National Diabetes Register (NDR). URL: https://www.ndr.nu/\#/english [accessed 2020-03-10]

20. Amcoff E, Edberg A, Enghardt Barbieri H, Lindroos AK, Nälsén C, Pearson M, et al. Riksmaten - Vuxna 2010-11 Livsmedelsoch Näringsintag Bland Vuxna i Sverige. Uppsala, Sweden: Livsmedelsverket; 2012. URL: https://www.livsmedelsverket.se/ globalassets/publikationsdatabas/rapporter/2011/riksmaten 2010 20111.pdf [accessed 2020-03-10]

21. Jeppsson J, Kobold U, Barr J, Finke A, Hoelzel W, Hoshino T, International Federation of Clinical Chemistry and Laboratory Medicine (IFCC). Approved IFCC reference method for the measurement of HbA1c in human blood. Clin Chem Lab Med 2002 Jan;40(1):78-89. [doi: 10.1515/CCLM.2002.016] [Medline: 11916276 ]

22. Landin-Olsson M, Jeppsson JO, Nordin G. HbA1c--New standardization introduced in Sweden. The new unit is mmol/mol [Article in Swedish]. Lakartidningen 2010 Dec 22;107(51-52):3282-3285. [Medline: 21294350]

23. Messerer M, Johansson S, Wolk A. The validity of questionnaire-based micronutrient intake estimates is increased by including dietary supplement use in Swedish men. J Nutr 2004 Jul;134(7):1800-1805. [doi: 10.1093/jn/134.7.1800] [Medline: 15226472]

24. Utdrag ur Indikatorbilagan för Nationella Riktlinjer för Sjukdomsförebyggande Metoder 2011 [Document in Swedish]. Stockholm, Sweden: Socialstyrelsen (The National Board of Health and Welfare); 2020 Feb. URL: https://www. socialstyrelsen.se/globalassets/sharepoint-dokument/artikelkatalog/ovrigt/2020-2-6596.pdf [accessed 2020-11-06]

25. Karlsson J, Persson LO, Sjöström L, Sullivan M. Psychometric properties and factor structure of the Three-Factor Eating Questionnaire (TFEQ) in obese men and women. Results from the Swedish Obese Subjects (SOS) study. Int J Obes Relat Metab Disord 2000 Dec;24(12):1715-1725. [doi: 10.1038/sj.ijo.0801442] [Medline: 11126230]

26. Bonn SE, Trolle Lagerros Y, Christensen SE, Möller E, Wright A, Sjölander A, et al. Active-Q: Validation of the web-based physical activity questionnaire using doubly labeled water. J Med Internet Res 2012 Feb 15;14(1):e29 [FREE Full text] [doi: 10.2196/jmir.1974] [Medline: 22356755]

27. Bonn SE, Bergman P, Trolle Lagerros Y, Sjölander A, Bälter K. A validation study of the web-based physical activity questionnaire Active-Q against the GENEA accelerometer. JMIR Res Protoc 2015 Jul 16;4(3):e86 [FREE Full text] [doi: 10.2196/resprot.3896] [Medline: 26183896]

28. Hays RD, Morales LS. The RAND-36 measure of health-related quality of life. Ann Med 2001 Jul;33(5):350-357. [doi: 10.3109/07853890109002089] [Medline: 11491194]

29. Scheier MF, Wrosch C, Baum A, Cohen S, Martire LM, Matthews KA, et al. The Life Engagement Test: Assessing purpose in life. J Behav Med 2006 Jun;29(3):291-298. [doi: 10.1007/s10865-005-9044-1] [Medline: 16565785]

30. Kecklund G, Åkerstedt T. The psychometric properties of the Karolinska Sleep Questionnaire. J Sleep Res 1992;1(1):113.

31. Akerstedt T, Knutsson A, Westerholm P, Theorell T, Alfredsson L, Kecklund G. Sleep disturbances, work stress and work hours: A cross-sectional study. J Psychosom Res 2002 Sep;53(3):741-748. [doi: 10.1016/s0022-3999(02)00333-1] [Medline: 12217447]

32. Cohen S, Kamarck T, Mermelstein R. A global measure of perceived stress. J Health Soc Behav 1983 Dec;24(4):385-396. [Medline: $\underline{6668417}$ ]

33. Amsberg S, Wredling R, Lins P, Adamson U, Johansson U. The psychometric properties of the Swedish version of the Problem Areas in Diabetes Scale (Swe-PAID-20): Scale development. Int J Nurs Stud 2008 Sep;45(9):1319-1328. [doi: 10.1016/j.ijnurstu.2007.09.010] [Medline: 17983618]

34. Eyler AA, Brownson RC, Donatelle RJ, King AC, Brown D, Sallis JF. Physical activity social support and middle- and older-aged minority women: Results from a US survey. Soc Sci Med 1999 Sep;49(6):781-789. [doi:

10.1016/s0277-9536(99)00137-9] [Medline: 10459889] 
35. Melin J, Bonn SE, Pendrill L, Trolle Lagerros Y. A questionnaire for assessing user satisfaction with mobile health apps: Development using Rasch measurement theory. JMIR Mhealth Uhealth 2020 May 26;8(5):e15909 [FREE Full text] [doi: 10.2196/15909] [Medline: $\underline{32452817]}$

36. Maderuelo-Fernandez JA, Recio-Rodríguez JI, Patino-Alonso MC, Pérez-Arechaederra D, Rodriguez-Sanchez E, Gomez-Marcos MA, et al. Effectiveness of interventions applicable to primary health care settings to promote Mediterranean diet or healthy eating adherence in adults: A systematic review. Prev Med 2015 Jul;76 Suppl:S39-S55. [doi: 10.1016/j.ypmed.2014.12.011] [Medline: 25524613]

37. Ley SH, Hamdy O, Mohan V, Hu FB. Prevention and management of type 2 diabetes: Dietary components and nutritional strategies. Lancet 2014 Jun 07;383(9933):1999-2007 [FREE Full text] [doi: 10.1016/S0140-6736(14)60613-9] [Medline: 24910231]

38. Forouhi NG, Misra A, Mohan V, Taylor R, Yancy W. Dietary and nutritional approaches for prevention and management of type 2 diabetes. BMJ 2018 Jun 13;361:k2234 [FREE Full text] [doi: 10.1136/bmj.k2234] [Medline: 29898883]

39. Holmen H, Torbjørnsen A, Wahl AK, Jenum AK, Småstuen MC, Arsand E, et al. A mobile health intervention for self-management and lifestyle change for persons with type 2 diabetes, Part 2: One-year results from the Norwegian randomized controlled trial RENEWING HEALTH. JMIR Mhealth Uhealth 2014 Dec 11;2(4):e57 [FREE Full text] [doi: 10.2196/mhealth.3882] [Medline: 25499872]

40. Tricco AC, Ivers NM, Grimshaw JM, Moher D, Turner L, Galipeau J, et al. Effectiveness of quality improvement strategies on the management of diabetes: A systematic review and meta-analysis. Lancet 2012 Jun 16;379(9833):2252-2261. [doi: 10.1016/S0140-6736(12)60480-2] [Medline: 22683130]

41. Evert AB, Dennison M, Gardner CD, Garvey WT, Lau KHK, MacLeod J, et al. Nutrition therapy for adults with diabetes or prediabetes: A consensus report. Diabetes Care 2019 May;42(5):731-754 [FREE Full text] [doi: 10.2337/dci19-0014] [Medline: 31000505]

\author{
Abbreviations \\ CONSORT: Consolidated Standards of Reporting Trials \\ CONSORT-EHEALTH: Consolidated Standards of Reporting Trials of Electronic and Mobile HEalth Applications \\ and onLine TeleHealth \\ CVD: cardiovascular disease \\ FFQ: food frequency questionnaire \\ HAPPY: Healthy eating using APP technologY \\ $\mathbf{H b A}_{1 \mathbf{c}}$ : glycated hemoglobin \\ HDL: high-density lipoprotein \\ IFCC: International Federation for Clinical Chemistry and Laboratory Medicine \\ LDL: low-density lipoprotein \\ mHealth: mobile health \\ SFO-V: Strategic Research Area Health Care Sciences
}

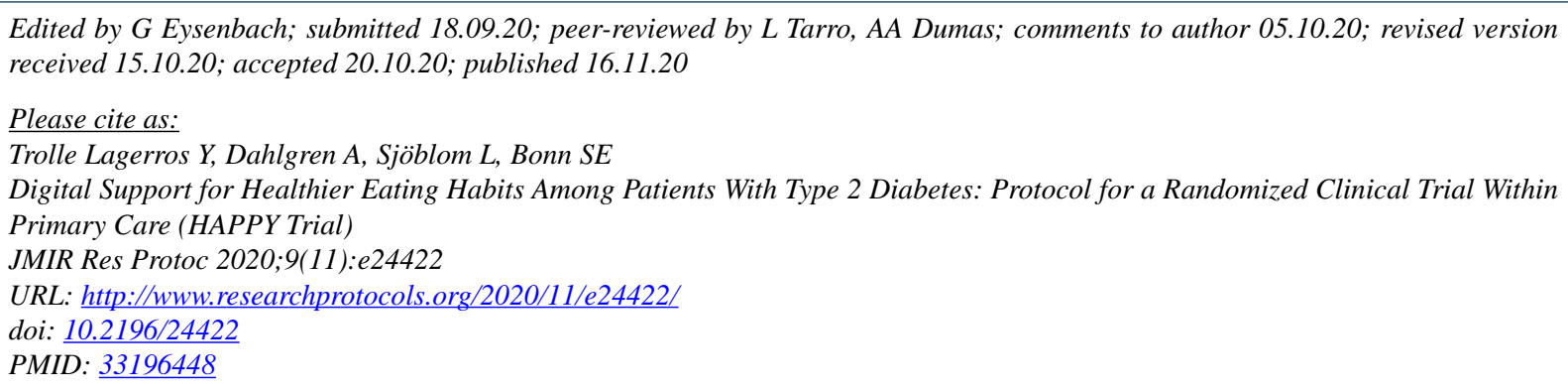

(CYlva Trolle Lagerros, Anna Dahlgren, Linnea Sjöblom, Stephanie E Bonn. Originally published in JMIR Research Protocols (http://www.researchprotocols.org), 16.11.2020. This is an open-access article distributed under the terms of the Creative Commons Attribution License (https://creativecommons.org/licenses/by/4.0/), which permits unrestricted use, distribution, and reproduction in any medium, provided the original work, first published in JMIR Research Protocols, is properly cited. The complete bibliographic information, a link to the original publication on http://www.researchprotocols.org, as well as this copyright and license information must be included. 